\title{
Viewpoint \\ Determining sensitivity to rapamycin and its analogues in breast cancer patients
}

\section{Caroline J Witton}

\author{
Endocrine Cancer Group, University of Glasgow, Glasgow, UK (currently seconded to DakoCytomation, Copenhagen, Denmark) \\ Corresponding author: Caroline Witton, cjw6r@clinmed.gla.ac.uk \\ Published: 17 December 2004
}

Breast Cancer Res 2005, 7:41-42 (DOI 10.1186/bcr985)

(C) 2004 BioMed Central Ltd

\section{Introduction}

Mammalian target of rapamycin (mTOR) is a kinase with sequence homology to phosphoinositol-3 kinase (PI3K). It is a downstream mediator in the PI3K/AKT pathway, which regulates proliferation, survival, mobility and angiogenesis. The targets of mTOR include p70s6 kinase and 4E-BP1 (for review, see Bjornsti and Houghton [1]). Rapamycin is an antibiotic and fungicide isolated from Streptomyces hygroscopicus that inhibits mTOR activity and has been approved as an immunosuppressive drug in organ transplant patients. Interest in rapamycin and analogues as cancer treatments is growing [2] because of the observation that the PI3K/AKT pathway is often altered in cancers. This can occur via mutation in the tumour suppressor gene PTEN, which downregulates the pathway, or over-expression of receptors (such as HER2) that stimulate the pathway. It can also occur via overexpression of other proteins in the pathway (such as AKT/protein kinase B) due to gene amplification or failure to break down the proteins.

Rapamycin itself has poor aqueous solubility and is not stable, and so several analogues (CCl-779, RAD001 and AP23573) have been developed that are being tested in clinical trials for cancer treatment. These new drugs can potentially be used for the treatment of breast cancer once those patients who will respond to the drug can be identified. In this review I summarize two recent papers that provide insight into the determinants of sensitivity to rapamycin and the potential synergism with conventional chemotherapies.

\section{Rapamycin and its analogues in breast cancer}

Study of breast cancer cell lines has suggested that cells that express the active (phosphorylated) form of AKT are sensitive to rapamycin treatment [3], as are those that are oestrogen receptor (ER) positive, lack PTEN, or over- express HER2 [4]. This result has been recapitulated in breast cancer patients, with Chan [4] reporting this year that patients who responded to CC1-779 in a clinical trial had lost PTEN expression and/or had HER2 overexpression. Although that trial was small, including only 109 patients, none of the 32 who were HER2 negative showed any significant response to treatment.

Two recent papers published in Clinical Cancer Research $[5,6]$ have tackled the subject of targeting mTOR with rapamycin in breast cancer. Zhou and coworkers [5] examined 165 primary breast cancers with antibodies directed against HER2 and phosphorylated forms of AKT, mTOR and 4E-BP1 (a downstream target of mTOR). They compared staining in the primary breast tumours with that seen in normal breast epithelium, fibroadenoma, intraductal hyperplasia and ductal carcinoma in situ. They found that phosphorylation levels of all three molecules generally increased during the transition from normal epithelium to invasive disease. None of the fibroadenomas expressed phosphorylated forms of the markers. Few of the normal breast samples expressed phosphorylated AKT (two out of eight with low expression) and phosphorylated mTOR (one out of eight with high expression), and none expressed phosphorylated 4E-BP1. The levels observed in intraductal hyperplasia and ductal carcinoma in situ were higher than in normal breast epithelium, and this trend continued with invasive carcinomas. As with in vitro studies, phosphorylated AKT/mTOR/4E-BP1 was more common in tumours that over-expressed HER2, and phosphorylated AKT was positively correlated with ER expression.

With univariate analysis those investigators also demonstrated that phosphorylated AKT, mTOR and 4EBP1 were associated with differences in disease-free survival (DFS). Patients whose tumours had high 
phosphorylated mTOR had significantly shorter DFS, whereas those with low phosphorylated AKT or 4E-BP1 had longer DFS. A multivariate analysis including conventional prognostic indicators was not presented, and so it is unclear whether this would add any information to current prognostic data. In a follow-up to this tissue-based study, they examined the effect of rapamycin on HER2-expressing breast cancer cell lines [5]. They concluded that HER2 over-expression leads to increased phosphorylated mTOR and phosphorylated 4EBP1 expression, and that these cells are sensitive to rapamycin treatment.

In the same issue of Clinical Cancer Research, Mondesire and coworkers [6] describe the interaction of rapamycin and standard chemotherapy agents in breast cancer cell lines and a xenograft mouse model of breast cancer. They observed that rapamycin alone resulted in growth inhibition of sensitive cell lines and that the effect of rapamycin in combination with paclitaxel, carboplatin and vinorelbine was synergistic (i.e. the combined effect is greater than that expected by the addition of the effects of each drug). Synergism was exhibited by increased growth inhibition in the case of paclitaxel and increased apoptosis when rapamycin was combined with both paclitaxel and carboplatin. These effects were only observed in rapamycin-sensitive cell lines and not in rapamycininsensitive ones. In addition, they found that use of rapamycin could increase the chemosensitivity of HER2 over-expressing cells that normally show some resistance to paclitaxel and carboplatin. Finally, they assessed the efficacy of rapamycin in combination with paclitaxel in nude mice with cell line xenografts. This experiment demonstrated that the combination was more effective in reducing tumour volume than either drug alone.

\section{Conclusion}

Both of these papers present evidence that activation of the AKT/mTOR pathway by HER2 (and possibly by other receptors such as ER) can lead to sensitivity of breast cancer cells to rapamycin and its analogues. Zhou and coworkers [5] showed that the active (phosphorylated) forms of proteins in the AKT/mTOR pathway can be detected in paraffin-embedded breast tumours using immunohistochemistry $(\mathrm{IHC})$ and that the information provided may have prognostic value. It is also expected to identify those patients with tumours that are sensitive to rapamycin and its analogues. In addition, the results presented by Mondesire and coworkers [6] suggest that, in those patients whose tumours are sensitive to rapamycin (or its analogues), the combination with conventional chemotherapy may have an even greater effect. However, it is not certain that phosphorylated forms of proteins can accurately and reliably be detected using conventional IHC on paraffin-embedded tissue. In a recent meeting presentation [7], concern was expressed that delayed fixation of samples could affect phosphorylation status of proteins and thus their detection.

In conclusion, these findings show promise that patients who will benefit from treatment with rapamycin or its analogues can be identified with IHC using antibodies against phosphorylated proteins. However, further work in this field is required to determine which target(s) are most useful for determining sensitivity and whether IHC is the best method to detect them.

\section{Competing interests}

The author(s) declare that they have no competing interests.

\section{References}

1. Bjornsti MA, Houghton PJ: The TOR pathway: a target for cancer therapy. Nat Rev Cancer 2004, 4:335-348.

2. Carraway $\mathrm{H}$, Hidalgo $\mathrm{M}$ : New targets for therapy in breast cancer: mammalian target of rapamycin (mTOR) antagonists. Breast Cancer Res 2004, 6:219-224.

3. Noh WC, Mondesire WH, Peng JY, Jian WG, Zhang HX, Dong JJ, Mills GB, Hung MC, Meric-Bernstam F: Determinants of rapamycin sensitivity in breast cancer cells. Clin Cancer Res 2004, 10:1013-1023.

4. Chan S: Targeting the mammalian target of rapamycin (mTOR): a new approach to treating cancer. Br J Cancer 2004 91:1420-1424.

5. Zhou X, Tan M, Stone HV, Klos KS, Lan KH, Yang Y, Yang W, Smith TL, Shi D, Yu D: Activation of the Akt/mammalian target of rapamycin/4E-BP1 pathway by ErbB2 overexpression predicts tumor progression in breast cancers. Clin Cancer Res 2004, 10:6779-6788.

6. Mondesire WH, Jian W, Zhang H, Ensor J, Hung MC, Mills GB, Meric-Bernstam F: Targeting mammalian target of rapamycin synergistically enhances chemotherapy-induced cytotoxicity in breast cancer cells. Clin Cancer Res 2004, 10:7031-7042.

7. Mohsin SK, Weiss H, Allred DC: Effects of formalin fixation on immunohistochemical expression of phosphorylated proteins in human breast cancer. In 26th Annual San Antonio Breast Cancer Symposium; 3-6 December 2003; San Antonio, TX. http://www.abstracts2view.com/bcs03/ 